\title{
Anti-Periodic Forced Oscillations of Damped Beams on Elastic Bearings
}

\author{
Sergiu Aizicovici and Michal Fečkan
}

Communicated by Charles Li, received June 14, 2004.

\begin{abstract}
We show the existence of anti-periodic solutions for certain damped linear beam equations with anti-periodic forcing terms and resting on nonlinear elastic bearings.
\end{abstract}

\section{Contents}

1. Introduction 339

2. Setting of the problem 340

3. Linear Equations 341

4. Nonlinear Equations $\quad 345$

5. Multivalued Equations 354

References $\quad 356$

\section{Introduction}

In this paper, we consider an anti-periodically forced and damped beam resting on two different bearings with purely elastic responses. The length of the beam is $\pi / 4$. The equation of vibrations is as follows

$$
\begin{aligned}
& u_{t t}+u_{x x x x}+\delta u_{t}+h_{1}(x, t)=0, \\
& u_{x x}(0, \cdot)=u_{x x}(\pi / 4, \cdot)=0, \\
& u_{x x x}(0, \cdot)=-f(u(0, \cdot))-h_{2}(t), \\
& u_{x x x}(\pi / 4, \cdot)=g(u(\pi / 4, \cdot))+h_{3}(t),
\end{aligned}
$$

where $u=u(x, t), \delta>0$ is a constant, $f, g \in C(\mathbb{R}, \mathbb{R})$ are odd functions and $h_{1} \in X$, $h_{2}, h_{3} \in Y$ are anti-periodic forcing terms. Here $X$ and $Y$ are the following Banach

1991 Mathematics Subject Classification. Primary 35B20; Secondary 47H10, 74K10.

Key words and phrases. Beam equations, anti-periodic solutions, topological degree.

The second author is partially supported by Grant GA-MS 1/6179/02. This paper was completed while the second author visited Ohio University. 
spaces

$$
\begin{aligned}
& X:=\{h \in C([0, \pi / 4] \times \mathbb{R}, \mathbb{R}) \mid h(x, t+T)=-h(x, t) \\
& \quad \text { for any }(x, t) \in[0, \pi / 4] \times \mathbb{R}\}, \\
& Y:=\{h \in C(\mathbb{R}, \mathbb{R}) \mid h(t+T)=-h(t) \text { for any } t \in \mathbb{R}\}
\end{aligned}
$$

endowed with the usual sup norms $\|\cdot\|$ for a fixed $T>0$.

Recently, we investigated the existence of periodic solutions of (1.1) for general non-odd functions $f, g$ and $T$-periodic $h_{1}(x, t)$ with $h_{2}(t)=h_{3}(t)=0$. In [9] and [10], we proved existence and non-existence results for $T$-periodic solutions of (1.1) depending on the forcing function $h_{1}(x, t)$. Chaotic solutions for equations similar to (1.1) are considered in $[2]$ and $[\mathbf{3}]$. The existence of free vibrations of undamped and unforced equations like (1.1) is studied in [8] and [11] by using variational methods.

Now we study the existence of anti-periodic (weak) solutions $u \in X$ of (1.1). The plan of the paper is as follows. In Section 2, we formulate the notion of a weak $T$-anti-periodic solution of (1.1). We also recall some well-known results on the corresponding linear eigenvalue problem. Then in Section 3, we study linear problems and certain Poincaré type inequalities related to (1.1). Section 4 contains the main existence result for weak $T$-anti-periodic solutions of (1.1), when in addition $h_{2}, h_{3} \in W^{1,2}(0, T)$. The approach relies on topological degree arguments. Some results are also presented for semilinear problems by assuming only $h_{2}, h_{3} \in Y$. In the final Section 5, we extend the main result (Theorem 4.2 ) of Section 4 to a discontinuous/multivalued case (cf. (5.2)). There we suppose that the functions $f$ and $g$ are upper semicontinuous with compact interval values.

Finally we note (cf. [11]) that equation (1.1) is a simple analogue of a more complicated shaft dynamics model introduced in [5] and [6].

\section{Setting of the problem}

By a weak $T$-anti-periodic solution of (1.1), we mean any $u \in X$ satisfying the identity

$$
\begin{aligned}
& \int_{0}^{T} \int_{0}^{\pi / 4}\left[u(x, t)\left\{v_{t t}(x, t)+v_{x x x x}(x, t)-\delta v_{t}(x, t)\right\}+h_{1}(x, t) v(x, t)\right] d x d t \\
& +\int_{0}^{T}\left\{\left(f(u(0, t))+h_{2}(t)\right) v(0, t)+\left(g(u(\pi / 4, t))+h_{3}(t)\right) v(\pi / 4, t)\right\} d t=0
\end{aligned}
$$

for any $v \in X^{\infty}$ with

$$
\begin{aligned}
X^{\infty}:= & \left\{v \in X \cap C^{\infty}([0, \pi / 4] \times \mathbb{R}) \mid v_{x x}(0, \cdot)=v_{x x}(\pi / 4, \cdot)\right. \\
& \left.=v_{x x x}(0, \cdot)=v_{x x x}(\pi / 4, \cdot)=0\right\}
\end{aligned}
$$

The eigenvalue problem

$$
\begin{aligned}
& w_{x x x x}(x)=\mu^{4} w(x), \\
& w_{x x}(0)=w_{x x}(\pi / 4)=0, \\
& w_{x x x}(0)=w_{x x x}(\pi / 4)=0
\end{aligned}
$$

is known $[\mathbf{1 1}]$ to possess a sequence of eigenvalues $\mu_{k}, k=-1,0,1, \cdots$ with

$$
\mu_{-1}=\mu_{0}=0
$$


and

$$
\cos \left(\mu_{k} \pi / 4\right) \cosh \left(\mu_{k} \pi / 4\right)=1, \quad k=1,2, \cdots .
$$

The corresponding orthonormal system of eigenvectors in $L^{2}(0, \pi / 4)$ is

$$
\begin{aligned}
& w_{-1}(x)=\frac{2}{\sqrt{\pi}}, \quad w_{0}(x)=\frac{16}{\pi}\left(x-\frac{\pi}{8}\right) \sqrt{\frac{3}{\pi}} \\
& w_{k}(x) \frac{4}{\sqrt{\pi} W_{k}}\left[\cosh \left(\mu_{k} x\right)+\cos \left(\mu_{k} x\right)\right. \\
& \left.-\frac{\cosh \xi_{k}-\cos \xi_{k}}{\sinh \xi_{k}-\sin \xi_{k}}\left(\sinh \left(\mu_{k} x\right)+\sin \left(\mu_{k} x\right)\right)\right]
\end{aligned}
$$

where the constants $W_{k}$ are given by the formulas

$$
W_{k}=\cosh \left(\xi_{k}\right)+\cos \xi_{k}-\frac{\cosh \xi_{k}-\cos \xi_{k}}{\sinh \xi_{k}-\sin \xi_{k}}\left(\sinh \xi_{k}+\sin \xi_{k}\right)
$$

for $\xi_{k}=\mu_{k} \pi / 4$. From (2.2) we get the asymptotic formulas

$$
1<\mu_{k}=2(2 k+1)+r(k) \quad \forall k \geq 1
$$

along with

$$
|r(k)| \leq \bar{c}_{1} e^{-\bar{c}_{2} k} \quad \forall k \geq 1,
$$

where $\bar{c}_{1}, \bar{c}_{2}$ are positive constants. Moreover, the eigenfunctions $\left\{w_{i}\right\}_{i=-1}^{\infty}$ are uniformly bounded in $C[0, \pi / 4]$.

\section{Linear Equations}

Let $H_{1} \in X, H_{2}, H_{3} \in Y$. In order to solve (2.1), we consider the equation

$$
\begin{aligned}
& \int_{0}^{T} \int_{0}^{\pi / 4}\left[u(x, t)\left\{v_{t t}(x, t)+v_{x x x x}(x, t)-\delta v_{t}(x, t)\right\}+H_{1}(x, t) v(x, t)\right] d x d t \\
& +\int_{0}^{T}\left\{H_{2}(t) v(0, t)+H_{3}(t) v(\pi / 4, t)\right\} d t=0
\end{aligned}
$$

for any $v \in X^{\infty}$. We look for $u(x, t)$ in the form

$$
u(x, t)=\sum_{i=-1}^{\infty} z_{i}(t) w_{i}(x) .
$$

We formally put (3.2) into (3.1) to get a system of ordinary differential equations

$$
\ddot{z}_{i}(t)+\delta \dot{z}_{i}(t)+\mu_{i}^{4} z_{i}(t)=h_{i}(t),
$$

where

$$
h_{i}(t)=-\left(\int_{0}^{\pi / 4} H_{1}(x, t) w_{i}(x) d x+H_{2}(t) w_{i}(0)+H_{3}(t) w_{i}(\pi / 4)\right) .
$$

Clearly $h_{i} \in Y$ for any $i \geq-1$. Since $\mu_{i}>0$ for $i \geq 1$, we reason as in [10] to conclude that equation (3.3) has a unique $T$-anti-periodic solution $z_{i} \in Y$, namely: 
(i): for $2 \mu_{i}^{2}>\delta, z_{i}$ is given by

$$
z_{i}(t)=\frac{2}{\bar{\omega}_{i}} \int_{-\infty}^{t} e^{-\delta(t-s) / 2} \sin \left(\frac{\bar{\omega}_{i}}{2}(t-s)\right) \times h_{i}(s) d s,
$$

where $\bar{\omega}_{i}=\sqrt{4 \mu_{i}^{4}-\delta^{2}}$;

(ii): for $2 \mu_{i}^{2}=\delta, z_{i}$ is given by

$$
z_{i}(t)=\int_{-\infty}^{t} e^{-\delta(t-s) / 2}(t-s) \times h_{i}(s) d s
$$

(iii): for $2 \mu_{i}^{2}<\delta, z_{i}$ is given by

$$
z_{i}(t)=\int_{-\infty}^{t} \frac{1}{\tilde{\omega}_{i}}\left(e^{\left(-\delta+\tilde{\omega}_{i}\right)(t-s) / 2}-e^{\left(-\delta-\tilde{\omega}_{i}\right)(t-s) / 2}\right) \times h_{i}(s) d s,
$$

where $\tilde{\omega}_{i}=\sqrt{\delta^{2}-4 \mu_{i}^{4}}$.

Like in [10], from (3.5)-(3.7) we get

$$
\begin{aligned}
& \left\|z_{i}\right\| \leq \frac{1}{\mu_{i}^{2}}\left(1+\frac{4}{\delta}\right)\left\|h_{i}\right\| \\
& \left\|\dot{z}_{i}\right\| \leq\left(\frac{4}{\delta}+\delta\right)\left\|h_{i}\right\|,
\end{aligned}
$$

for any $i \geq 1$. Since $\mu_{i}=0$ for $i=-1$ and $i=0$, we see that (3.3) has a unique solution $z_{i} \in Y$ for $i=-1,0$ on $[0, T]$ given by

$$
\begin{aligned}
& z_{i}(t)=\frac{1}{\delta} \int_{0}^{t} h_{i}(s) d s-\frac{1}{2 \delta} \int_{0}^{T} h_{i}(s) d s \\
& -\frac{1}{\delta} \int_{0}^{t} h_{i}(s) h_{i}(s) d s+\frac{\int_{0}^{T} e^{-\delta(T+t-s)} h_{i}(s) d s}{\delta\left(1+e^{-\delta T}\right.},
\end{aligned}
$$

which yields

$$
\begin{aligned}
& \left\|z_{i}\right\| \leq\left(\frac{3 T}{2 \delta}+\frac{3}{\delta^{2}}\right)\left\|h_{i}\right\|, \\
& \left\|\dot{z}_{i}\right\| \leq \frac{2}{\delta}\left\|h_{i}\right\| .
\end{aligned}
$$

From (3.4) we get

$$
\left\|h_{i}\right\| \leq M_{1}\left(\frac{\pi}{4}\left\|H_{1}\right\|+\left\|H_{2}\right\|+\left\|H_{3}\right\|\right)
$$

for

$$
M_{1}:=\sup _{i \geq 1, x}\left|w_{i}(x)\right|
$$


Plugging (3.5)-(3.7) into (3.2) and using (3.8)-(3.10), we obtain

$$
\begin{aligned}
& \|u\| \leq \sum_{i=-1}^{\infty}\left\|z_{i}\right\|\left\|w_{i}\right\| \leq M_{1}^{2}\left\{\frac{3 T}{\delta}+\frac{6}{\delta^{2}}\right. \\
& \left.+\left(1+\frac{4}{\delta}\right) \sum_{i=1}^{\infty} \frac{1}{\mu_{i}^{2}}\right\}\left(\frac{\pi}{4}\left\|H_{1}\right\|+\left\|H_{2}\right\|+\left\|H_{3}\right\|\right) \\
& \leq M_{2}\left(\left\|H_{1}\right\|+\left\|H_{2}\right\|+\left\|H_{3}\right\|\right)
\end{aligned}
$$

for

$$
M_{2}:=M_{1}^{2}\left\{\frac{3 T}{\delta}+\frac{6}{\delta^{2}}+\left(1+\frac{4}{\delta}\right) \sum_{i=1}^{\infty} \frac{1}{\mu_{i}^{2}}\right\}
$$

We note that $\sum_{i=1}^{\infty} \frac{1}{\mu_{i}^{2}}<\infty$. Summarizing the above results, we arrive at:

Proposition 3.1. For any given functions $H_{1} \in X, H_{2}, H_{3} \in Y$, there is a unique solution $L\left(H_{1}, H_{2}, H_{3}\right):=u(x, t) \in X$ of equation (3.1). The linear mapping $L: X \times Y \times Y \rightarrow X$ is compact with the norm $\|L\| \leq M_{2}$ when the norm on $V:=X \times Y \times Y$ is given by $\|v\|:=\left\|H_{1}\right\|+\left\|H_{2}\right\|+\left\|H_{3}\right\|, v=\left(H_{1}, H_{2}, H_{3}\right) \in V$.

Now let us fix $n \in \mathbb{N}$ and consider an approximating linear problem to (3.1), namely

$$
\begin{aligned}
& \int_{0}^{T} \int_{0}^{\pi / 4}\left[u_{n}(x, t)\left\{v_{t t}(x, t)+v_{x x x x}(x, t)-\delta v_{t}(x, t)\right\}+H_{1}(x, t) v(x, t)\right] d x d t \\
& +\int_{0}^{T}\left\{H_{2}(t) v(0, t)+H_{3}(t) v(\pi / 4, t)\right\} d t=0
\end{aligned}
$$

for any $v \in X_{n}^{\infty}$ with

$$
X_{n}^{\infty}:=\left\{v \in X^{\infty} \mid v(x, t)=\sum_{i=-1}^{n} v_{i}(t) w_{i}(x) \quad \text { for } \quad v_{i} \in Y \cap C^{\infty}(\mathbb{R}, \mathbb{R})\right\} .
$$

Here wee look for $u_{n}(x, t)$ in the form

$$
u_{n}(x, t)=\sum_{i=-1}^{n} z_{i}(t) w_{i}(x) .
$$

By repeating the above approach to (3.1) for (3.11), we arrive at the following result.

Proposition 3.2. For any given functions $H_{1} \in X, H_{2}, H_{3} \in Y$, equation (3.11) has a unique solution $u_{n} \in X$ of the form

$$
u_{n}(x, t)=\sum_{i=-1}^{n} z_{i}(t) w_{i}(x) .
$$

Such a solution satisfies the following conditions:

(a):

$$
\begin{aligned}
& \max _{-1 \leq i \leq n}\left\|z_{i}\right\|\left(i^{2}+1\right) \leq M_{3}\left(\left\|H_{1}\right\|+\left\|H_{2}\right\|+\left\|H_{3}\right\|\right) \\
& \max _{-1 \leq i \leq n}\left\|\dot{z}_{i}\right\| \leq M_{3}\left(\left\|H_{1}\right\|+\left\|H_{2}\right\|+\left\|H_{3}\right\|\right)
\end{aligned}
$$


for

$$
M_{3}:=\sup _{i \geq 1}\left\{\frac{i^{2}+1}{\mu_{i}^{2}}\right\}\left(1+\frac{4}{\delta}\right)+\frac{3 T}{\delta}+\frac{6}{\delta^{2}}+\frac{4}{\delta}+\delta .
$$

(b): The linear mapping $L_{n}: X \times Y \times Y \rightarrow X$ defined by $L_{n}\left(H_{1}, H_{2}, H_{3}\right):=$ $u_{n}(x, t)$ is compact.

Now we recall the following result from $[\mathbf{1 0}]$ :

Proposition 3.3. A sequence $\left\{u_{n}\right\}_{n=1}^{\infty} \subset X$ is precompact if there is a constant $M>0$ such that

$$
\sup _{i \geq-1, n \geq 1}\left\|z_{i, n}\right\|\left(i^{2}+1\right)<M, \quad \sup _{i \geq-1, n \geq 1}\left\|\dot{z}_{i, n}\right\|<M,
$$

where $u_{n}(x, t)=\sum_{i=-1}^{\infty} z_{i, n}(t) w_{i}(x)$.

We end this section with two Poincaré inequalities.

Proposition 3.4. The following Poincaré inequality holds

$$
\|w\| \leq \frac{\pi^{3 / 2}}{8 \sqrt{105}} \sqrt{\int_{0}^{\pi / 4} w_{x x}(x)^{2} d x}
$$

for any $w \in W^{2,2}(0, \pi / 4)$ satisfying

$$
\int_{0}^{\pi / 4} w(x) d x=\int_{0}^{\pi / 4} x w(x) d x=0 .
$$

Proof of Proposition 3.4. For any $h \in L^{2}(0, \pi / 4)$, the solution $w(x)$ of the differential equation

$$
w_{x x}(x)=h(x)
$$

which satisfies conditions (3.12) is given by

$$
w(x)=\int_{0}^{\pi / 4} G(x, s) h(s) d s
$$

for a Green function $G$ defined by

$$
G(x, s)=\left\{\begin{array}{l}
\left(\frac{48}{\pi^{2}} s^{2}-\frac{128}{\pi^{3}} s^{3}\right) x+\frac{16}{\pi^{2}} s^{3}-\frac{8}{\pi} s^{2} \quad \text { for } \quad 0 \leq s \leq x \leq \frac{\pi}{4} \\
\left(\frac{48}{\pi^{2}} s^{2}-\frac{128}{\pi^{3}} s^{3}-1\right) x+\frac{16}{\pi^{2}} s^{3}-\frac{8}{\pi} s^{2}+s \quad \text { for } \quad 0 \leq x \leq s \leq \frac{\pi}{4} .
\end{array}\right.
$$

Thus for any $x \in[0, \pi / 4]$ we have

$$
|w(x)| \leq \max _{x \in[0, \pi / 4]} \sqrt{\int_{0}^{\pi / 4} G(x, s)^{2} d s} \sqrt{\int_{0}^{\pi / 4} h(s)^{2} d s} .
$$


By using Mathematica, we compute

$$
\begin{gathered}
\int_{0}^{\pi / 4} G(x, s)^{2} d s=\frac{1}{6720 \pi^{3}}\left(\pi^{6}-44 \pi^{5} x+624 \pi^{4} x^{2}-2240 \pi^{3} x^{3}\right. \\
\left.-8960 \pi^{2} x^{4}+64512 \pi x^{5}-86016 x^{6}\right),
\end{gathered}
$$

and check that the maximum of the right-hand side of (3.13) on the interval $[0, \pi / 4]$ is $\pi^{3} / 6720$, which is attained at the end points $x=0$ and $x=\pi / 4$. Consequently, we obtain

$$
|w(x)| \leq \max _{x \in[0, \pi / 4]} \sqrt{\int_{0}^{\pi / 4} G(x, s)^{2} d s} \sqrt{\int_{0}^{\pi / 4} h(s)^{2} d s} \leq \frac{\pi^{3 / 2}}{8 \sqrt{105}} \sqrt{\int_{0}^{\pi / 4} h(s)^{2} d s .}
$$

The proof is complete.

Proposition 3.5. Let $\widetilde{X}$ be a Banach space with a norm $|\cdot|$. Then the following Poincaré inequality holds

$$
\max _{t \in[0, T]}|h(t)| \leq \sqrt{T} \sqrt{\int_{0}^{T}|\dot{h}(t)|^{2} d t}
$$

for any $T$-anti-periodic function $h \in W^{1,2}(0, T ; \widetilde{X})$.

For the proof of Proposition 3.5, see [1].

\section{Nonlinear Equations}

First, we suppose that in addition to the conditions listed in the Introduction, $h_{2}, h_{3} \in W^{1,2}(0, T)$. Now we use the Bubnov-Galerkin approximation method. So we put the form

$$
u_{n}(x, t)=\sum_{i=-1}^{n} z_{i}(t) w_{i}(x)
$$

into (1.1) to derive the system of ordinary differential equations

$$
\begin{aligned}
& \ddot{z}_{i}(t)+\delta \dot{z}_{i}(t)+\mu_{i}^{4} z_{i}(t)+h_{1, i}(t) \\
& +f\left(\sum_{i=-1}^{n} z_{i}(t) w_{i}(0)\right) w_{i}(0)+g\left(\sum_{i=-1}^{n} z_{i}(t) w_{i}(\pi / 4)\right) w_{i}(\pi / 4) \\
& +h_{2}(t) w_{i}(0)+h_{3}(t) w_{i}(\pi / 4)=0,
\end{aligned}
$$

where

$$
h(x, t)=\sum_{i=-1}^{\infty} h_{1, i}(t) w_{i}(x) .
$$

The system (4.2) is a Bubnov-Galerkin approximation of (1.1). Now we solve (4.2). For this purpose, we consider a Banach space $Z_{n}=Y^{n+2}$ with the norm

$$
\|z\|_{n}:=\left\|u_{n}\right\|
$$


where $u_{n}(x, t)$ is defined by (4.1) for $z=\left(z_{-1}(t), z_{0}(t), z_{1}(t), \cdots, z_{n}(t)\right) \in Z_{n}$. Next we introduce the following nonlinear operator

$$
\begin{aligned}
& F_{n}: Z_{n} \rightarrow Z_{n} \\
& F_{n}(z):=\left(\widetilde{z}_{-1}(t), \widetilde{z}_{0}(t), \widetilde{z}_{1}(t), \cdots, \widetilde{z}_{n}(t)\right) \\
& \sum_{i=-1}^{n} \widetilde{z}_{i}(t) w_{i}(x):=L_{n}\left(h_{1}, f\left(\sum_{i=-1}^{n} z_{i}(t) w_{i}(0)\right)+h_{2}(t), g\left(\sum_{i=-1}^{n} z_{i}(t) w_{i}(\pi / 4)\right)+h_{3}(t)\right) \\
& z=\left(z_{-1}(t), z_{0}(t), z_{1}(t), \cdots, z_{n}(t)\right) .
\end{aligned}
$$

We note that according to Proposition 3.2, the operator $F_{n}$ is compact. Then (4.2) is equivalent to the fixed point problem

$$
z=F_{n}(z) .
$$

In order to solve (4.3) uniformly for $n \in \mathbb{N}$, by the Leray-Schauder degree theory for maps [4], [12], it is enough to show that there is a constant $c_{1}>0$ such that for any $\lambda \geq 1$ and $n \in \mathbb{N}$, every solution of the equation

$$
\lambda z=F_{n}(z)
$$

satisfies $\|z\|_{n} \leq c_{1}$. But this means that we must find an a-priori bound for the $T$-anti-periodic solutions of the system

$$
\begin{aligned}
& \lambda \ddot{z}_{i}(t)+\lambda \delta \dot{z}_{i}(t)+\lambda \mu_{i}^{4} z_{i}(t)+h_{1, i}(t) \\
& +f\left(\sum_{i=-1}^{n} z_{i}(t) w_{i}(0)\right) w_{i}(0)+g\left(\sum_{i=-1}^{n} z_{i}(t) w_{i}(\pi / 4)\right) w_{i}(\pi / 4) \\
& +h_{2}(t) w_{i}(0)+h_{3}(t) w_{i}(\pi / 4)=0,
\end{aligned}
$$

for any $\lambda \geq 1$.

To do this, we first multiply $(4.5)$ by $\dot{z}_{i}(t)$, integrate the result from 0 to $T$, and then sum up these equations to obtain

$$
\begin{aligned}
& \sum_{i=-1}^{n} \lambda \int_{0}^{T} \ddot{z}_{i}(t) \dot{z}_{i}(t) d t+\sum_{i=-1}^{n} \lambda \delta \int_{0}^{T} \dot{z}_{i}(t)^{2} d t+\lambda \sum_{i=-1}^{n} \mu_{i}^{4} \int_{0}^{T} z_{i}(t) \dot{z}_{i}(t) d t \\
& +\int_{0}^{T} f\left(\sum_{i=-1}^{n} z_{i}(t) w_{i}(0)\right)\left(\sum_{i=-1}^{n} \dot{z}_{i}(t) w_{i}(0)\right) d t+ \\
& \int_{0}^{T} g\left(\sum_{i=-1}^{n} z_{i}(t) w_{i}(\pi / 4)\right)\left(\sum_{i=-1}^{n} \dot{z}_{i}(t) w_{i}(\pi / 4)\right) d t \\
& +\int_{0}^{T} \sum_{i=-1}^{n} h_{1, i}(t) \dot{z}_{i}(t) d t+\int_{0}^{n} \sum_{i=-1}^{n} h_{2}(t) \dot{z}_{i}(t) w_{i}(0) d t+ \\
& \int_{0}^{T} \sum_{i=-1}^{n} h_{3}(t) \dot{z}_{i}(t) w_{i}(\pi / 4) d t=0 .
\end{aligned}
$$

This implies

$$
\begin{aligned}
& \lambda \delta \sum_{i=-1}^{n} \int_{0}^{T} \dot{z}_{i}(t)^{2} d t+\int_{0}^{T} \sum_{i=-1}^{n} h_{1, i}(t) \dot{z}_{i}(t) d t \\
& -\sum_{i=-1}^{n} \int_{0}^{T} \dot{h}_{2}(t) z_{i}(t) w_{i}(0) d t-\sum_{i=-1}^{n} \int_{0}^{T} \dot{h}_{3}(t) z_{i}(t) w_{i}(\pi / 4) d t=0
\end{aligned}
$$


and hence

$$
\begin{aligned}
& \lambda \delta \sum_{i=-1}^{n} \int_{0}^{T} \dot{z}_{i}(t)^{2} d t \leq \sqrt{\int_{0}^{T} \sum_{i=-1}^{n} h_{1, i}(t)^{2}} d t \sqrt{\int_{0}^{T} \sum_{i=-1}^{n} \dot{z}_{i}(t)^{2} d t+} \\
& +M_{1}\left(\sqrt{\int_{0}^{T} \dot{h}_{2}(t)^{2} d t}+\sqrt{\int_{0}^{T} \dot{h}_{3}(t)^{2} d t}\right) \sqrt{2+\sum_{i=1}^{n} \frac{1}{\mu_{i}^{4}}} \\
& \sqrt{\int_{0}^{T}\left(z_{-1}(t)^{2}+z_{0}(t)^{2}+\sum_{i=1}^{n} \mu_{i}^{4} z_{i}(t)^{2}\right) d t} \\
& \begin{array}{l}
\leq \widetilde{K}\left(\sqrt{\int_{0}^{T} \sum_{i=-1}^{n} \dot{z}_{i}(t)^{2} d t}+\sqrt{\int_{0}^{T}\left(z_{-1}(t)^{2}+z_{0}(t)^{2}+\sum_{i=1}^{n} \mu_{i}^{4} z_{i}(t)^{2}\right) d t}\right) \\
\leq \widetilde{K}\left(\sqrt{\int_{0}^{T} \sum_{i=-1}^{n} \dot{z}_{i}(t)^{2} d t}+\sqrt{\int_{0}^{T}\left(z_{-1}(t)^{2}+z_{0}(t)^{2}\right) d t}+\sqrt{\int_{0}^{T} \sum_{i=1}^{n} \mu_{i}^{4} z_{i}(t)^{2} d t}\right) .
\end{array}
\end{aligned}
$$

The Poincaré inequality of Proposition 3.5 gives

$$
\lambda \delta \sum_{i=-1}^{n} \int_{0}^{T} \dot{z}_{i}(t)^{2} d t \leq \widetilde{K}\left((1+T) \sqrt{\int_{0}^{T} \sum_{i=-1}^{n} \dot{z}_{i}(t)^{2} d t}+\sqrt{\int_{0}^{T} \sum_{i=1}^{n} \mu_{i}^{4} z_{i}(t)^{2} d t}\right)
$$

Next, we multiply (4.5) by $z_{i}(t)$, integrate from 0 to $T$, and then sum up these equations to arrive at

$$
\begin{aligned}
& \sum_{i=-1}^{n} \lambda \int_{0}^{T} \ddot{z}_{i}(t) z_{i}(t) d t+\sum_{i=-1}^{n} \lambda \delta \int_{0}^{T} \dot{z}_{i}(t) z_{i}(t) d t+\lambda \sum_{i=-1}^{n} \mu_{i}^{4} \int_{0}^{T} z_{i}(t)^{2} d t \\
& +\int_{0}^{T} f\left(\sum_{i=-1}^{n} z_{i}(t) w_{i}(0)\right)\left(\sum_{i=-1}^{n} z_{i}(t) w_{i}(0)\right) d t+ \\
& \int_{0}^{T} g\left(\sum_{i=-1}^{n} z_{i}(t) w_{i}(\pi / 4)\right)\left(\sum_{i=-1}^{n} z_{i}(t) w_{i}(\pi / 4)\right) d t \\
& +\int_{0}^{T} \sum_{i=-1}^{T} h_{1, i}(t) z_{i}(t) d t+\int_{0}^{T} \sum_{i=-1}^{n} h_{2}(t) z_{i}(t) w_{i}(0) d t+ \\
& \int_{0}^{T} \sum_{i=-1}^{n} h_{3}(t) z_{i}(t) w_{i}(\pi / 4) d t=0 .
\end{aligned}
$$


This gives

$$
\begin{aligned}
& \lambda \sum_{i=-1}^{n} \mu_{i}^{4} \int_{0}^{T} z_{i}(t)^{2} d t=\lambda \sum_{i=-1}^{n} \int_{0}^{T} \dot{z}_{i}(t)^{2} d t \\
& -\int_{0}^{T} f\left(\sum_{i=-1}^{n} z_{i}(t) w_{i}(0)\right)\left(\sum_{i=-1}^{n} z_{i}(t) w_{i}(0)\right) d t- \\
& \int_{0}^{T} g\left(\sum_{i=-1}^{n} z_{i}(t) w_{i}(\pi / 4)\right)\left(\sum_{i=-1}^{n} z_{i}(t) w_{i}(\pi / 4)\right) d t \\
& -\int_{0}^{T} \sum_{i=-1}^{n} h_{1, i}(t) z_{i}(t) d t-\int_{0}^{T} \sum_{i=-1}^{n} h_{2}(t) z_{i}(t) w_{i}(0) d t-\int_{0}^{T} \sum_{i=-1}^{n} h_{3}(t) z_{i}(t) w_{i}(\pi / 4) d t .
\end{aligned}
$$

We impose the following condition

- (H) There are non-negative constants $\alpha_{f}$ and $\alpha_{g}$, with

$$
\left(\alpha_{f}+\alpha_{g}\right) M_{1}\left(2+\sum_{i=1}^{\infty} \frac{1}{\mu_{i}^{4}}\right)<1
$$

and a non-negative constant $\beta$ such that

$$
f(u) u \geq-\alpha_{f} u^{2}-\beta, \quad g(u) u \geq-\alpha_{g} u^{2}-\beta
$$

for any $u \in \mathbb{R}$. 
As a result, we obtain

$$
\begin{aligned}
& \begin{array}{l}
\sum_{i=-1}^{n} \mu_{i}^{4} \int_{0}^{T} z_{i}(t)^{2} d t \leq \lambda \sum_{i=-1}^{n} \int_{0}^{T} \dot{z}_{i}(t)^{2} d t \\
+\sqrt{\int_{0}^{T} \sum_{i=-1}^{n} h_{1, i}(t)^{2} d t \sqrt{\int_{0}^{T} \sum_{i=-1}^{n} z_{i}(t)^{2} d t}+2 \beta}
\end{array} \\
& +\left(\alpha_{f}+\alpha_{g}\right) M_{1}\left(2+\sum_{i=1}^{\infty} \frac{1}{\mu_{i}^{4}}\right)\left(T^{2} \sum_{i=-1}^{n} \int_{0}^{T} \dot{z}_{i}(t)^{2} d t+\int_{0}^{T} \sum_{i=1}^{n} \mu_{i}^{4} z_{i}(t)^{2} d t\right) \\
& +M_{1}\left(\sqrt{\int_{0}^{T} h_{2}(t)^{2} d t}+\sqrt{\int_{0}^{T} h_{3}(t)^{2} d t}\right) \sqrt{2+\sum_{i=1}^{n} \frac{1}{\mu_{i}^{4}}} \\
& \times \sqrt{\int_{0}^{T}\left(z_{-1}(t)^{2}+z_{0}(t)^{2}+\sum_{i=1}^{n} \mu_{i}^{4} z_{i}(t)^{2}\right) d t} \\
& \leq \bar{K}_{1}\left((\lambda+1) \sum_{i=-1}^{n} \int_{0}^{T} \dot{z}_{i}(t)^{2} d t+\sqrt{\sum_{i=-1}^{n} \int_{0}^{T} \dot{z}_{i}(t)^{2} d t}+1\right. \\
& +\sqrt{\left.\int_{0}^{T} \sum_{i=1}^{n} \mu_{i}^{4} z_{i}(t)^{2} d t\right)}+\left(\alpha_{f}+\alpha_{g}\right) M_{1}\left(2+\sum_{i=1}^{\infty} \frac{1}{\mu_{i}^{4}}\right) \int_{0}^{T} \sum_{i=1}^{n} \mu_{i}^{4} z_{i}(t)^{2} d t \\
& \leq \bar{K}\left(\lambda \sum_{i=-1}^{n} \int_{0}^{T} \dot{z}_{i}(t)^{2} d t+\sqrt{\sum_{i=-1}^{n} \int_{0}^{T} \dot{z}_{i}(t)^{2} d t}+1\right. \\
& \left.+\sqrt{\int_{0}^{T} \sum_{i=1}^{n} \mu_{i}^{4} z_{i}(t)^{2} d t}\right)+\left(\alpha_{f}+\alpha_{g}\right) M_{1}\left(2+\sum_{i=1}^{\infty} \frac{1}{\mu_{i}^{4}}\right) \int_{0}^{T} \sum_{i=1}^{n} \mu_{i}^{4} z_{i}(t)^{2} d t .
\end{aligned}
$$

Here $\bar{K}_{1}$ and $\bar{K}$ are positive constants which are independent of $z_{i}(t)$ and $n$. By denoting

$$
A_{n}:=\sqrt{\sum_{i=-1}^{n} \int_{0}^{T} \dot{z}_{i}(t)^{2} d t}, \quad B_{n}:=\sqrt{\int_{0}^{T} \sum_{i=1}^{n} \mu_{i}^{4} z_{i}(t)^{2} d t},
$$

inequalities (4.6) and (4.9) take the forms

$$
\begin{aligned}
& \lambda A_{n}^{2} \leq \hat{K}\left(A_{n}+B_{n}\right) \\
& B_{n}^{2} \leq \hat{K}\left(\lambda A_{n}^{2}+A_{n}+1+B_{n}\right)+\left(\alpha_{f}+\alpha_{g}\right) M_{1}\left(2+\sum_{i=1}^{\infty} \frac{1}{\mu_{i}^{4}}\right) B_{n}^{2}
\end{aligned}
$$

for a constant $\hat{K}$ depending on the constants $\alpha_{f}, \alpha_{g}, \beta, \delta, T$ and functions $h_{1}(x, t)$, $h_{2}(t), h_{3}(t)$. By putting

$$
\gamma:=1-\left(\alpha_{f}+\alpha_{g}\right) M_{1}\left(2+\sum_{i=1}^{\infty} \frac{1}{\mu_{i}^{4}}\right)
$$


from (4.10) we get

$$
\begin{aligned}
& \lambda A_{n}^{2} \leq \hat{K}\left(A_{n}+B_{n}\right) \\
& B_{n}^{2} \leq \frac{\hat{K}}{\gamma}\left(\lambda A_{n}^{2}+A_{n}+1+B_{n}\right) .
\end{aligned}
$$

Now, (4.11) implies

$$
\begin{aligned}
& A_{n}^{2} \leq \hat{K}\left(A_{n}+B_{n}\right) \\
& B_{n}^{2} \leq\left(\frac{\hat{K}}{\gamma}+\frac{\hat{K}^{2}}{\gamma}\right)\left(A_{n}+B_{n}\right)+\frac{\hat{K}}{\gamma}
\end{aligned}
$$

and then

$$
\left(A_{n}+B_{n}\right)^{2} / 2 \leq A_{n}^{2}+B_{n}^{2} \leq\left(\hat{K}+\frac{\hat{K}}{\gamma}+\frac{\hat{K}^{2}}{\gamma}\right)\left(A_{n}+B_{n}\right)+\frac{\hat{K}}{\gamma},
$$

which gives

$$
A_{n}+B_{n} \leq \Gamma
$$

for

$$
\Gamma:=\left(\hat{K}+\frac{\hat{K}}{\gamma}+\frac{\hat{K}^{2}}{\gamma}\right)+\sqrt{\left(\hat{K}+\frac{\hat{K}}{\gamma}+\frac{\hat{K}^{2}}{\gamma}\right)^{2}+\frac{2 \hat{K}}{\gamma}} .
$$

Now from Section 2, we immediately obtain

$$
\int_{0}^{\pi / 4} w_{i, x x}(x)^{2} d x=\mu_{i}^{4}, \quad \int_{0}^{\pi / 4} w_{i, x x}(x) w_{j, x x}(x) d x=0 \quad \text { for } \quad i \neq j .
$$

Then (4.1), (4.12) and (4.13) imply

$$
\int_{0}^{\pi / 4} u_{n, x x}(x, t)^{2} d x=\sum_{i=1}^{n} \mu_{i}^{4} z_{i}(t)^{2} \leq \Gamma^{2}
$$

for any $t \in \mathbb{R}$. Hence the Poincaré inequality of Proposition 3.4 gives

$$
\left|\bar{u}_{n}(x, t)\right| \leq \frac{\pi^{3 / 2}}{8 \sqrt{105}} \Gamma
$$

for any $(x, t) \in[0, \pi / 4] \times \mathbb{R}$ and

$$
\bar{u}_{n}(x, t)=\sum_{i=1}^{n} z_{i}(t) w_{i}(x) .
$$

On the other hand, the Poincaré inequality of Proposition 3.5 and estimate (4.12) imply

$$
\left|z_{-1}(t) w_{-1}(x)+z_{0}(t) w_{0}(x)\right| \leq 2 M_{1} \sqrt{T} \Gamma .
$$

Consequently, estimates (4.14) and (4.15) give

$$
\left\|u_{n}\right\| \leq \Theta:=\left(2 M_{1} \sqrt{T}+\frac{\pi^{3 / 2}}{8 \sqrt{105}}\right) \Gamma .
$$

Summarizing, we obtain the following result. 
Proposition 4.1. For any $n \geq 1$, every solution

$$
u_{n}(x, t)=\sum_{i=-1}^{n} z_{i}(t) w_{i}(x)
$$

of (4.2) satisfies (4.16).

Now returning to equations (4.3) and (4.4), and applying Proposition 4.1, we obtain the next theorem.

THEOREM 4.1. For any $n \geq 1$, there is a solution

$$
u_{n}(x, t)=\sum_{i=-1}^{n} z_{i}(t) w_{i}(x)
$$

of (4.2) satisfying (4.16).

Remark that

$$
\begin{aligned}
& \left|h_{1, i}(t)\right|=\left|\int_{0}^{\pi / 4} h_{1}(x, t) w_{i}(x) d x\right| \leq \frac{\pi}{4}\left\|h_{1}\right\| M_{1}, \\
& \left|f\left(\sum_{i=-1}^{n} z_{i}(t) w_{i}(0)\right) w_{i}(0)\right|+\left|g\left(\sum_{i=-1}^{n} z_{i}(t) w_{i}(\pi / 4)\right) w_{i}(\pi / 4)\right| \leq 2 K_{4} M_{1},
\end{aligned}
$$

for

$$
K_{4}:=\max _{|z| \leq \Theta}\{|f(z)|,|g(z)|\} .
$$

Then Proposition 3.2 ensures the existence of a constant $K_{5}>0$ such that the solutions

$$
u_{n}(x, t)=\sum_{i=-1}^{n} z_{i, n}(t) w_{i}(x)
$$

of (4.2) from Theorem 4.1 satisfy

$$
\sup _{i \geq-1, n \geq 1}\left\|z_{i, n}\right\|\left(i^{2}+1\right) \leq K_{5}, \quad \sup _{i \geq-1, n \geq 1}\left\|\dot{z}_{i, n}\right\| \leq K_{5}
$$

Then according to Proposition 3.3, there is a subsequence $\left\{u_{n_{i}}(x, t)\right\}_{i=1}^{\infty}$ of $\left\{u_{n}(x, t)\right\}_{n=1}^{\infty}$ which is uniformly convergent to a function $u \in X$ on $[0, \pi / 4] \times[0, T]$. On the other hand, equation (4.2) implies that $u_{n}(x, t)$ solves the following approximating equation

$$
\begin{aligned}
& \int_{0}^{T} \int_{0}^{\pi / 4}\left[u_{n}(x, t)\left\{v_{t t}(x, t)+v_{x x x x}(x, t)-\delta v_{t}(x, t)\right\}+h_{1}(x, t) v(x, t)\right] d x d t \\
& +\int_{0}^{T}\left\{\left(f\left(u_{n}(0, t)\right)+h_{2}(t)\right) v(0, t)+\left(g\left(u_{n}(\pi / 4, t)\right)+h_{3}(t)\right) v(\pi / 4, t)\right\} d t=0
\end{aligned}
$$

for any $v \in X_{n}^{\infty}$. But then clearly the limit function $u(x, t)$ satisfies (2.1) for any $v \in X_{n}^{\infty}$ and any $n \in \mathbb{N}$. Since $\cup_{n \in \mathbb{N}} X_{n}^{\infty}$ is dense in $X^{\infty}$ with respect to the topology of $X$, we see that $u(x, t)$ satisfies (2.1) for any $v \in X^{\infty}$; in other words, $u$ is a weak solution of (1.1). Summarizing, we obtain the following result:

THEOREM 4.2. If $f, g \in C(\mathbb{R}, \mathbb{R})$ are odd functions satisfying condition $(H)$, and $h_{1} \in X, h_{2}, h_{3} \in Y \cap W^{1,2}(0, T)$, then equation (1.1) possesses a weak $T$-antiperiodic solution. 
Theorem 4.2 is an improvement of results in $[\mathbf{9}]$ and [10], since we assume in [9] that the function $h_{1}(x, t)$ is small $T$-periodic, while in [10], we consider only functions $f(u)$ and $g(u)$ with at most linear growth at infinity. We also studied (1.1) in [9] and [10] with $h_{2}(t)=h_{3}(t)=0$. Of course, now both functions $f(u)$ and $g(u)$ are odd and the forcing terms are $T$-anti-periodic. For instance, condition $(\mathrm{H})$ holds (see inequalities (4.8)) if

$$
\liminf _{u \rightarrow+\infty} f(u)+\liminf _{u \rightarrow+\infty} g(u)>-\infty .
$$

As an example, if

$$
f(u)=f_{1} u+f_{2} u^{3}, \quad g(u)=g_{1} u+g_{2} u^{3}
$$

for constants $f_{1}, f_{2}, g_{1}$ and $g_{2}$, then (4.17) holds if $f_{2}>0$ and $g_{2}>0$, so that both springs are hard at the ends of the beam.

Now let us consider the case when both functions $f(u)$ and $g(u)$ are linear, i.e.

$$
f(u)=f_{1} u, \quad g(u)=g_{1} u .
$$

Then Theorem 4.2 is applicable if

$$
\left(\max \left\{-f_{1}, 0\right\}+\max \left\{-g_{1}, 0\right\}\right) M_{1}\left(2+\sum_{i=1}^{\infty} \frac{1}{\mu_{i}^{4}}\right)<1 .
$$

Then by using Proposition 3.1, we can rewrite (2.1) as the linear equation

$$
M u=h_{4}
$$

for

$$
\begin{aligned}
& M u=u-L\left(0, f_{1} u(0, \cdot), g_{1} u(\pi / 4, \cdot)\right), \\
& h_{4}=L\left(h_{1}, h_{2}, h_{3}\right) .
\end{aligned}
$$

Clearly $M$ is a linear bounded Fredholm operator from $X$ to $X$ with index 0 . Theorem 4.2 ensures that $X^{\infty} \subset R(M)$ - the range of $M$. Indeed, for any $v \in X^{\infty}$, we take $h_{1}(x, t)=-v_{t t}-v_{x x x x}-\delta v_{t}, h_{2}(t)=0$ and $h_{3}(t)=0$. Then $h_{4}=$ $L\left(h_{1}, 0,0\right)=v(x, t)$. On the other hand, Theorem 4.2 implies the existence of $u \in X$ such that $M(u)=h_{4}$. Hence $v \in R(M)$, i.e. $X^{\infty} \subset R(M)$. Since $M$ is of Fredholm type, $R(M)$ is closed. Since $X^{\infty}$ is dense in $X$ and $X^{\infty} \subset R(M)$, we get $R(M)=X$ and then $N(M)=\{0\}$ - the kernel of $M$. Consequently, $M$ is a linear isomorphism from $X$ to $X$. So we obtain the following result.

THEOREM 4.3. Let $f(u)=f_{1} u$ and $g(u)=g_{1} u$ for constants $f_{1}, g_{1}$ satisfying (4.18). Then equation (1.1) possesses a unique weak T-anti-periodic solution $\widetilde{L}\left(h_{1}, h_{2}, h_{3}\right):=u \in X$ for any $h_{1} \in X$ and $h_{2}, h_{3} \in Y$. In addition, the linear mapping $\widetilde{L}: X \times Y \times Y \rightarrow X$ is compact.

The implicit function theorem together with Theorem 4.3 yields:

Theorem 4.4. If $f, g \in C^{1}(\mathbb{R}, \mathbb{R})$ are odd functions and $f_{1}=f^{\prime}(0), g_{1}=$ $g^{\prime}(0)$ satisfy (4.18), then there are positive constants $K_{1}, \varepsilon_{0}$ such that for any given functions $h_{1} \in X, h_{2}, h_{3} \in Y$ with $\left\|h_{1}\right\|+\left\|h_{2}\right\|+\left\|h_{3}\right\|<\varepsilon_{0}$, equation (1.1) possesses a unique small weak $T$-anti-periodic solution $u \in X$ satisfying $\|u\| \leq$ $K_{1}\left(\left\|h_{1}\right\|+\left\|h_{2}\right\|+\left\|h_{3}\right\|\right)$.

Furthermore, by using Schauder's fixed point theorem [4] along with Theorem 4.3 and adapting the arguments of [10], we obtain the following result. 
THEOREM 4.5. Let $f(u)=f_{1} u+\widetilde{f}(u)$ and $g(u)=g_{1} u+\widetilde{g}(u)$ with odd functions $\widetilde{f}, \widetilde{g} \in C(\mathbb{R}, \mathbb{R})$ and constants $f_{1}, g_{1}$ satisfying (4.18). If there are positive constants $c_{11}, c_{12}, c_{21}, c_{22}$ where

$$
c_{12}+c_{22}<1 /\|\widetilde{L}\|
$$

and such that

$$
\begin{aligned}
& |\widetilde{f}(u)| \leq c_{11}+c_{12}|u|, \quad \forall u \in \mathbb{R} \\
& |\widetilde{g}(u)| \leq c_{21}+c_{22}|u|, \quad \forall u \in \mathbb{R},
\end{aligned}
$$

then for any given functions $h_{1} \in X, h_{2}, h_{3} \in Y$, equation (1.1) possesses a weak $T$-anti-periodic solution $u \in X$.

Of course, when $\tilde{f}, \widetilde{g}$ have sublinear growth at infinity:

$$
\lim _{|u| \rightarrow \infty} \tilde{f}(u) / u=0, \quad \lim _{|u| \rightarrow \infty} \tilde{g}(u) / u=0,
$$

then the assumptions of Theorem 4.5 hold and equation (1.1) possesses a weak $T$-anti-periodic solution $u \in C\left([0, \pi / 4] \times S^{T}\right)$ for any $h_{1} \in X, h_{2}, h_{3} \in Y$.

Theorems 4.3, 4.4 and 4.5 are improvements of similar results in [10]. We note that in Theorem 4.2 we have more general odd functions $f, g$ than in Theorems 4.3, $4.4,4.5$, but on the other hand, we suppose in Theorem 4.2 that $h_{2}, h_{3} \in W^{1,2}(0, T)$.

Finally, we numerically estimate from above the constant

$$
M_{1}\left(2+\sum_{i=1}^{\infty} \frac{1}{\mu_{i}^{4}}\right)
$$

from condition $(\mathrm{H})$. We know from $[\mathbf{3}]$ that

$$
M_{1} \leq 4.763953413 \text {. }
$$

Now we evaluate the sum

$$
\sum_{i=1}^{\infty} \frac{1}{\xi_{i}^{4}}
$$

We have from $[\mathbf{3}]$ that

$$
\left|\xi_{i}-\frac{\pi(2 i+1)}{2}\right| \leq \frac{e^{-\pi i}}{2}
$$

for any $i \geq 1$. Since $\xi_{i} \geq 4$ and $\pi(2 i+1) / 2 \geq 4$ for all $i \geq 1$, we have

$$
\left|\frac{1}{\xi_{i}^{4}}-\frac{16}{\pi^{4}(2 i+1)^{4}}\right| \leq \frac{1}{256}\left|\xi_{i}-\frac{\pi(2 i+1)}{2}\right| \leq \frac{e^{-\pi i}}{512} .
$$

By solving the equations

$$
\cos \xi_{i} \cosh \left(\xi_{i}\right)=1, \quad i=1,2, \cdots
$$

with the help of Mathematica, we get

$$
\begin{aligned}
& \xi_{1}=4.730040744, \quad \xi_{2}=7.853204624 \\
& \xi_{3}=10.995607838, \quad \xi_{4}=14.137165491 \\
& \xi_{5}=17.278759657, \quad \xi_{6}=20.420352245
\end{aligned}
$$

By using

$$
\sum_{i=0}^{\infty} \frac{1}{(2 i+1)^{4}}=\frac{\pi^{4}}{96}
$$


(4.21) and (4.22), we obtain

$$
\begin{aligned}
& \sum_{i=1}^{\infty} \frac{1}{\xi_{i}^{4}} \leq \sum_{i=1}^{6} \frac{1}{\xi_{i}^{4}}+\sum_{i=7}^{\infty}\left|\frac{1}{\xi_{i}^{4}}-\frac{16}{\pi^{4}(2 i+1)^{4}}\right|+\sum_{i=1}^{\infty} \frac{16}{\pi^{4}(2 i+1)^{4}} \\
& -\sum_{i=1}^{6} \frac{16}{\pi^{4}(2 i+1)^{4}} \leq \sum_{i=1}^{6} \frac{1}{\xi_{i}^{4}}+\frac{e^{-7 \pi}}{512\left(1-e^{-\pi}\right)}+\frac{1}{6}-\frac{16}{\pi^{4}} \\
& -\frac{16}{\pi^{4}} \sum_{i=1}^{6} \frac{1}{(2 i+1)^{4}}=0.002381090 .
\end{aligned}
$$

Consequently, from (4.20) and (4.23), we derive

$$
M_{1}\left(2+\sum_{i=1}^{\infty} \frac{1}{\mu_{i}^{4}}\right)=M_{1}\left(2+\frac{\pi^{4}}{256} \sum_{i=1}^{\infty} \frac{1}{\xi_{i}^{4}}\right) \leq 9.532223039 .
$$

Hence, the inequality (4.7) of condition $(\mathrm{H})$ holds, if

$$
\alpha_{f}+\alpha_{g}<1 / 9.532223039=0.104907322
$$

and similarly, the inequality (4.18) holds, if

$$
\left(\max \left\{-f_{1}, 0\right\}+\max \left\{-g_{1}, 0\right\}\right)<0.104907322 .
$$

\section{Multivalued Equations}

In this section, we study (1.1) when $f$ and $g$ are multivalued, i.e., we suppose:

: (C1) $f, g: \mathbb{R} \rightarrow 2^{\mathbb{R}} \backslash \emptyset$ are odd and upper semicontinuous mappings with compact interval values.

: (C2) There are non-negative constants $\alpha_{f}$ and $\alpha_{g}$ satisfying (4.7), and a non-negative constant $\beta$ such that

$$
\begin{aligned}
& v u \geq-\alpha_{f} u^{2}-\beta, \quad \forall v \in f(u) \\
& v u \geq-\alpha_{g} u^{2}-\beta, \quad \forall v \in g(u)
\end{aligned}
$$

for any $u \in \mathbb{R}$.

REMARK 5.1. i) According to [7], condition (C1) is equivalent to the existence of lower semicontinuous functions $f_{-}, g_{-}: \mathbb{R} \rightarrow \mathbb{R}$ and upper semicontinuous functions $f_{+}, g_{+}: \mathbb{R} \rightarrow \mathbb{R}$ such that

$$
\begin{aligned}
& f_{-}(u) \leq f_{+}(u), \quad g_{-}(u) \leq g_{+}(u), \\
& f(u)=\left[f_{-}(u), f_{+}(u)\right], \quad g(u)=\left[g_{-}(u), g_{+}(u)\right]
\end{aligned}
$$

for any $u \in \mathbb{R}$. Moreover, the oddness of $f$ and $g$ implies

$$
-f_{+}(u)=f_{-}(-u), \quad-g_{+}(u)=g_{-}(-u)
$$

for any $u \in \mathbb{R}$.

ii) Since (5.1) holds, condition (C2) is equivalent to the assumption that the functions $f_{-}$and $g_{-}$satisfy condition $(\mathrm{H})$ with constants $\alpha_{f}, \alpha_{g}$ and $\beta$, respectively.

Now the equation of vibrations is as follows

$$
\begin{aligned}
& u_{t t}+u_{x x x x}+\delta u_{t}+h_{1}(x, t)=0, \\
& u_{x x}(0, \cdot)=u_{x x}(\pi / 4, \cdot)=0, \\
& u_{x x x}(0, \cdot) \in-f(u(0, \cdot))-h_{2}(t), \\
& u_{x x x}(\pi / 4, \cdot) \in g(u(\pi / 4, \cdot))+h_{3}(t) .
\end{aligned}
$$


By a weak $T$-anti-periodic solution of (5.2), we mean any $u \in X$ satisfying the identity

$$
\begin{aligned}
& \int_{0}^{T} \int_{0}^{\pi / 4}\left[u(x, t)\left\{v_{t t}(x, t)+v_{x x x x}(x, t)-\delta v_{t}(x, t)\right\}+h_{1}(x, t) v(x, t)\right] d x d t \\
& +\int_{0}^{T}\left\{f_{1}(t) v(0, t)+g_{1}(t) v(\pi / 4, t)\right\} d t=0
\end{aligned}
$$

for any $v \in X^{\infty}$ and some $f_{1}, g_{1} \in L^{2}(0, T)$ with

$$
\begin{aligned}
& f_{1}(t) \in f(u(0, t))+h_{2}(t), \\
& g_{1}(t) \in g(u(\pi / 4, t))+h_{3}(t)
\end{aligned}
$$

for a.a. $t \in(0, T)$.

We take $\widetilde{\alpha}_{f}=\alpha_{f_{-}}, \widetilde{\alpha}_{g}=\alpha_{g_{-}}$and $\widetilde{\beta}=2 \beta$ and we find the corresponding constant $\Theta$ from (4.16) for these $\widetilde{\alpha}_{f}, \widetilde{\alpha}_{g}$ and $\widetilde{\beta}$. Then it is not difficult to modify the proof of Proposition 1.1 (d) of [7], p. 7 to show that for any $\varepsilon, 0<\varepsilon<1 / 2$ there are continuous and odd functions

$$
f_{\varepsilon}, g_{\varepsilon}:[-\Theta-1, \Theta+1] \rightarrow \mathbb{R}
$$

such that

$$
\begin{aligned}
& f_{\varepsilon}(u) \in f((u-\varepsilon, u+\varepsilon))+(-\varepsilon, \varepsilon), \\
& g_{\varepsilon}(u) \in g((u-\varepsilon, u+\varepsilon))+(-\varepsilon, \varepsilon)
\end{aligned}
$$

for any $u \in[-\Theta-1, \Theta+1]$. Then there is a small $\varepsilon_{0}>0$ such that for any $0<\varepsilon<\varepsilon_{0}$, the functions $f_{\varepsilon}(u)$ and $g_{\varepsilon}(u)$ satisfy condition $(\mathrm{H})$ with constants $\widetilde{\alpha}_{f}$, $\widetilde{\alpha}_{g}$ and $\widetilde{\beta}$ on $[-\Theta-1, \Theta+1]$. Now we extend $f_{\varepsilon}$ and $g_{\varepsilon}$ to the whole $\mathbb{R}$ so that they are continuous and odd, and they satisfy condition $(\mathrm{H})$ with constants $\widetilde{\alpha}_{f}, \widetilde{\alpha}_{g}$ and $\widetilde{\beta}$ on $\mathbb{R}$. Then we apply Theorem 4.2 to get a function $u_{\varepsilon} \in X$ satisfying

$$
\begin{aligned}
& \int_{0}^{T} \int_{0}^{\pi / 4}\left[u_{\varepsilon}(x, t)\left\{v_{t t}(x, t)+v_{x x x x}(x, t)-\delta v_{t}(x, t)\right\}+h_{1}(x, t) v(x, t)\right] d x d t \\
& +\int_{0}^{T}\left\{\left(f_{\varepsilon}\left(u_{\varepsilon}(0, t)\right)+h_{2}(t)\right) v(0, t)+\left(g_{\varepsilon}\left(u_{\varepsilon}(\pi / 4, t)\right)+h_{3}(t)\right) v(\pi / 4, t)\right\} d t=0
\end{aligned}
$$

for any $v \in X^{\infty}$. We note that according to the choice of the constant $\Theta, u_{\varepsilon}(x, t)$ satisfies (4.16), so it is a solution for nonextended $f_{\varepsilon}$ and $g_{\varepsilon}$. For this reason, (5.5) holds. Moreover, we know that the sequence $\left\{u_{\varepsilon}\right\}_{0<\varepsilon<\varepsilon_{0}}$ is precompact in $X$. Then $\sup _{0<\varepsilon<\varepsilon_{0}}\left\|u_{\varepsilon}\right\|<\infty$. From (5.4) and (C1) we see that $\sup _{0<\varepsilon<\varepsilon_{0}}\left\|f_{\varepsilon}\left(u_{\varepsilon}\right)\right\|<\infty$. So the sequences $\left\{f_{\varepsilon}\left(u_{\varepsilon}(0, \cdot)\right)\right\}_{0<\varepsilon<\varepsilon_{0}}$ and $\left\{g_{\varepsilon}\left(u_{\varepsilon}(\pi / 4, \cdot)\right)\right\}_{0<\varepsilon<\varepsilon_{0}}$ are bounded in $L^{2}(0, T)$. Summarizing, we can find a subsequence $\left\{u_{\varepsilon_{i}}\right\}_{i=1}^{\infty}$ of $\left\{u_{\varepsilon}\right\}_{0<\varepsilon<\varepsilon_{0}}$ such that

$$
\begin{aligned}
& \varepsilon_{i} \rightarrow 0, \\
& u_{\varepsilon_{i}} \rightarrow u \text { in } X, \\
& f_{\varepsilon_{i}}\left(u_{\varepsilon_{i}}(0, \cdot)\right) \rightarrow f_{0}(t) \quad \text { weakly in } L^{2}(0, T), \\
& g_{\varepsilon_{i}}\left(u_{\varepsilon_{i}}(\pi / 4, \cdot)\right) \rightarrow g_{0}(t) \quad \text { weakly in } L^{2}(0, T)
\end{aligned}
$$

as $i \rightarrow \infty$ for some $u \in X$ and $f_{0}, g_{0} \in L^{2}(0, T)$. Now we take $\zeta>0$. Then the upper semicontinuity of $f$ and $g$, and (5.4) imply that there is an $i_{0}$ such that for 
any $i>i_{0}$, one has

$$
\begin{aligned}
& f_{\varepsilon_{i}}\left(u_{\varepsilon_{i}}(0, \cdot)\right) \in f(u(0, t))+[-\zeta, \zeta] \\
& g_{\varepsilon_{i}}\left(u_{\varepsilon_{i}}(\pi / 4, \cdot)\right) \in g(u(\pi / 4, t))+[-\zeta, \zeta]
\end{aligned}
$$

for any $t \in[0, T]$. On the other hand, it is obvious that the sets

$$
\left\{\begin{array}{l}
\left.s \in L^{2}(0, T) \mid s(t) \in f(u(0, t))+[-\zeta, \zeta] \quad \text { for a.a. } t \in(0, T)\right\} \\
\left.s \in L^{2}(0, T) \mid s(t) \in g(u(\pi / 4, t))+[-\zeta, \zeta] \text { for a.a. } t \in(0, T)\right\}
\end{array}\right.
$$

are closed and convex in the Hilbert space $L^{2}(0, T)$. Consequently, they are also weakly closed, so that

$$
\begin{aligned}
& f_{0}(t) \in f(u(0, t))+[-\zeta, \zeta], \\
& g_{0}(t) \in g(u(\pi / 4, t))+[-\zeta, \zeta]
\end{aligned}
$$

for a.a. $t \in(0, T)$. Since $\zeta>0$ is arbitrarily small and condition $(\mathrm{C} 1)$ holds, from (5.8) we get

$$
f_{0}(t) \in f(u(0, t)), \quad g_{0}(t) \in g(u(\pi / 4, t))
$$

for a.a. $t \in(0, T)$.

Now by passing to the limit as $i \rightarrow \infty$ with $\varepsilon=\varepsilon_{i}$ in (5.5) for a fixed $v \in X^{\infty}$, we see that the function $u(x, t)$ from (5.6) satisfies (5.3) with

$$
f_{1}(t)=f_{0}(t)+h_{2}(t), \quad g_{1}(t)=g_{0}(t)+h_{3}(t)
$$

for any $v \in X^{\infty}$. Hence such $u(x, t)$ is a weak $T$-anti-periodic solution of (5.2). Summarizing, we obtain the following result:

Theorem 5.1. If $f, g: \mathbb{R} \rightarrow 2^{\mathbb{R}} \backslash \emptyset$ satisfy conditions $(C 1),(C 2)$ and $h_{1} \in$ $X, h_{2}, h_{3} \in Y \cap W^{1,2}(0, T)$, then equation (5.2) possesses a weak $T$-anti-periodic solution.

Theorem 5.1 is certainly applicable to the simplest multivalued mappings $f(u)=$ $g(u)=\operatorname{sgn}(u)($ cf. [7]) with

$$
\operatorname{sgn}(u)= \begin{cases}-1 & \text { for } u<0 \\ {[-1,1]} & \text { for } u=0 \\ 1 & \text { for } u>0\end{cases}
$$

The multivalued problem (5.2) was not studied in the papers mentioned in the Introduction.

\section{References}

[1] AIZICOVICI, S. and PAVEL, N. H. Anti-periodic solutions to a class of nonlinear differential equations in Hilbert space, J. Funct. Anal. 99 (1991), 387-408.

[2] BATTELLI, F. and FEČKAN, M. Chaos in the beam equation, preprint (2003).

[3] BATTELLI, F. and FEČKAN, M. Homoclinic orbits of slowly periodically forced and weakly damped beams resting on weakly elastic bearings, Adv. Differential Equations 8 (2003), 1043-1080.

[4] BERGER, M. S. Nonlinearity and Functional Analysis, Academic Press, New York 1977.

[5] CAPRIZ, G. Self-excited vibrations of rotors, Int. Union Theor. Appl. Mech., Symp. Lyngby/Denmark 1974, Springer-Verlag 1975.

[6] CAPRIZ, G. and LARATTA, A. Large amplitude whirls of rotors, Vibrations in Rotating Machinery, Churchill College, Cambridge 1976.

[7] DEIMLING, K. Multivalued Differential Equations, W. De Gruyter, Berlin 1992.

[8] FEČKAN, M. Free vibrations of beams on bearings with nonlinear elastic responses, J. Differential Equations 154 (1999), 55-72. 
[9] FEČKAN, M. Periodically forced damped beams resting on nonlinear elastic bearings, Mathematica Slovaca (to appear).

[10] FEČKAN, M. Forced oscillations of beams on elastic bearings, Electr. J. Qualitative Theor. Differential Equations (to appear)

[11] FEIREISEL, E. Nonzero time periodic solutions to an equation of Petrovsky type with nonlinear boundary conditions: Slow oscillations of beams on elastic bearings, Ann. Scuola Norm. Sup. Pisa 20 (1993), 133-146.

[12] MAWHIN, J. Equivalence theorems for nonlinear operator equations and coincidence degree theory for some mappings in locally convex topological vector spaces, J. Differential Equations 12 (1972), 610-636.

Department of Mathematics, Ohio University, Athens, OH 45701, USA

E-mail address: aizicovi@bing.math.ohiou.edu

Department of Mathematical Analysis, Comenius University, Mlynská dolina, 842 48 Bratislava, Slovakia

E-mail address: michal.feckan@fmph.uniba.sk 